\title{
STUDY OF COMPLICATIONS IN SURGICAL MANAGEMENT OF METACARPAL AND PHALANGEAL FRACTURES
}

Hemanth H. P1, Abhijith Patil2, Darshan M. S3, Ponnilan J4

${ }^{1}$ Senior Resident, Department of Orthopaedics, Bangalore Medical College and Research Institute.

${ }^{2}$ Senior Resident, Department of Orthopaedics, Bangalore Medical College and Research Institute.

3 Junior Resident, Department of Orthopaedics, Bangalore Medical College and Research Institute.

4Junior Resident, Department of Orthopaedics, Bangalore Medical College and Research Institute.

\section{ABSTRACT}

\section{BACKGROUND AND OBJECTIVES}

Hand fractures if not treated properly will result in deformity, stiffness and loss of function leading to functional deformity. Fracture healing in hand is not an isolated goal, rather it is the functional result that is of paramount importance. Recent studies have shown good functional results with surgical treatment of metacarpal fractures using K-wires and mini-external fixators as compared to the conservative treatment.

\section{METHODS}

This is a prospective study of 60 cases of metacarpal and phalangeal fractures treated surgically with mini-external fixators and K-wires, admitted to Bowring and Lady Curzon Hospital and Victoria Hospital attached to Bangalore Medical College and Research Institute from Oct. 2013 to Sep. 2015.

\section{RESULTS}

We followed up all the patients and the duration ranged from 12 weeks to 20 weeks (Mean 16 weeks). All fractures eventually united. We had totally 7 complications, which included superficial infection.(1), stiffness of MCP joints.(1), non-union.(2), mal-union.(2) and gangrene.(2) We had no case of any purely implant related complications like K-wire migration or pin-tract infection. Fifth and fourth metacarpals were most commonly involved in our series. Out of 60 patients 33 patients (54.54\%) had excellent result, 19 patients (31.81\%) had good result and 8 patients (13.63\%) had poor result.

\section{INTERPRETATION AND CONCLUSION}

Displaced metacarpal fractures treated surgically by mini-external fixator. K-wire gives excellent result in terms of union, recovery of daily activities and stiffness. There will be fewer chances of infection and early bone union in case of CRIF with K-wire than ORIF with K-wire. However, stiffness of hand joints, especially metacarpo-phalangeal joint is of major concern and physiotherapy plays a major role to combat stiffness.

\section{KEYWORDS}

Fracture, Metacarpal, Phalanges.

HOW TO CITE THIS ARTICLE: Hemanth HP, Patil A, Darshan MS, et al. Study of complications in surgical management of metacarpal and phalangeal fractures. J. Evolution Med. Dent. Sci. 2016;5(45):2817-2819, DOI: 10.14260/jemds/2016/658

\section{INTRODUCTION}

To prevent the complications of open reduction and internal fixation like infection, soft tissue trauma, etc., and with the advent of image intensifier CRIF with K-wire percutaneously under image intensifier has given better results, faster healing, faster return of daily activities and faster rehabilitation. Early and appropriate physiotherapy other than accurate reduction and fixation affect recovery of hand mobility and function. In our study we are dealing with patients with metacarpal fractures treated with mini external fixators, ORIF with Kwire, CRIF with K-wire percutaneously under image intensifier.

\section{OBJECTIVE}

To study the technical difficulties and complications of metacarpal and phalangeal fractures treated surgically.

Financial or Other, Competing Interest: None.

Submission 03-05-2016, Peer Review 16-05-2016,

Acceptance 21-05-2016, Published 06-06-2016.

Corresponding Author:

Dr. Hemanth H. P,

D1-402, L \& T South City,

Arakere, Mico Layout,

Bangalore-560076.

E-mail: drhemanthhp@gmail.com

DOI: $10.14260 /$ jemds $/ 2016 / 658$

\section{Inclusion Criteria}

1. Closed fractures of phalanges and metacarpals.

2. Type 1 , type 2 , type $3 a$, type $3 b$ open fractures.

\section{Exclusion Criteria}

1. Type 3C open fractures.

2. Chronic immunocompromised state, such as Diabetes mellitus.

\section{MATERIAL AND METHODS}

Metacarpal fractures are reduced by closed methods under Carm or by open methods and are internally fixed by K-wires. Out of total 60 cases of metacarpal fractures, 40 cases were treated with K-wire of which 32 were closed reduced under Carm guidance, 8 were opened, 4 cases were treated with external fixators, and remaining cases were managed conservatively. Reduction was achieved by dorsal pressure over the fracture site and a dorsal push on the metacarpal head on the flexed digit. After reduction of fracture, Kirschner wire was inserted into the metacarpal head through the metacarpophalangeal joint, which was held in full flexion. When K-wire was well across the fracture site, wrist was flexed and wire insertion continued until the wire made its exit dorsally distal to the wrist. 
Then K-wire was extracted proximally until its distal end was out of head of the metacarpal, metacarpo-phalangeal joint is put through full range of motion confirming the withdrawal of $\mathrm{K}$-wire, then K-wire is cut at the distal end and it is bent to prevent proximal migration. Proper rotational alignment was determined in relationship to the other fingers by flexing other fingers to see that it points to the scaphoid tubercle. Some unstable oblique fracture of a PP can be treated by CR and percutaneous pinning with a $\mathrm{K}$-wire inserted across the fracture. The fracture is reduced by longitudinal traction and compressed with a towel clip, while the K-wires $(1-2.5 \mathrm{~mm})$ are drilled transversely across the fracture ? When possible, fractured finger should be flexed fully at all the joints and compared with the adjacent uninjured fingers.

A small hand-held power driver aids in accurate drilling and placement of the K-wires. The location and fracture pattern dictate the size, number and path of the K-wires. $\mathrm{K}$ wire is inserted mid-laterally to avoid injury to the extensor hood and the flexor tendon. One oblique K-wire usually is preferable to two crossed wires because the former allows some impaction at the fracture, whereas the latter causes distraction. After insertion, the wires are bent and left outside to aid in removal after fracture union as an outpatient procedure. Mini-external fixator: For displaced and comminuted fractures; application of mini external-fixation device is the preferred treatment.

\section{PROCEDURE}

Two transverse pins are placed proximal and distal to the fracture and inserted to the mid-axial or dorsolateral incisions. Pre-drilling is done bi-cortically with $1.5 \mathrm{~mm}$ power drill bit and $2 \mathrm{~mm}$ pin is inserted. The connecting rods and swivel clamps are inserted and tightened. Alternatively, the wires can be externally stabilized by a segment of polymethylmethacrylate.

\begin{tabular}{|c|c|c|}
\hline Excellent & Good & Poor \\
\hline $\begin{array}{c}\text { Pain-free } \\
\text { union }\end{array}$ & Pain-free union & Pain or non-union \\
\hline No deformity & $\begin{array}{c}\text { Minimal } \\
\text { deformity }\end{array}$ & $\begin{array}{c}\text { Deformity affecting } \\
\text { function }\end{array}$ \\
\hline TAM $>215^{\circ}$ & TAM $<180^{\circ}$ & TAM $>180^{\circ}$ \\
\hline $\begin{array}{c}\text { PIP motion }> \\
100^{\circ}\end{array}$ & PIP motion $>80^{\circ}$ & PIP motion $<80^{\circ}$ \\
\hline \multicolumn{3}{|c|}{$\begin{array}{c}\text { Table 1: Belsky's Criteria for } \\
\text { Assessment of Injured Finger }\end{array}$} \\
\hline
\end{tabular}

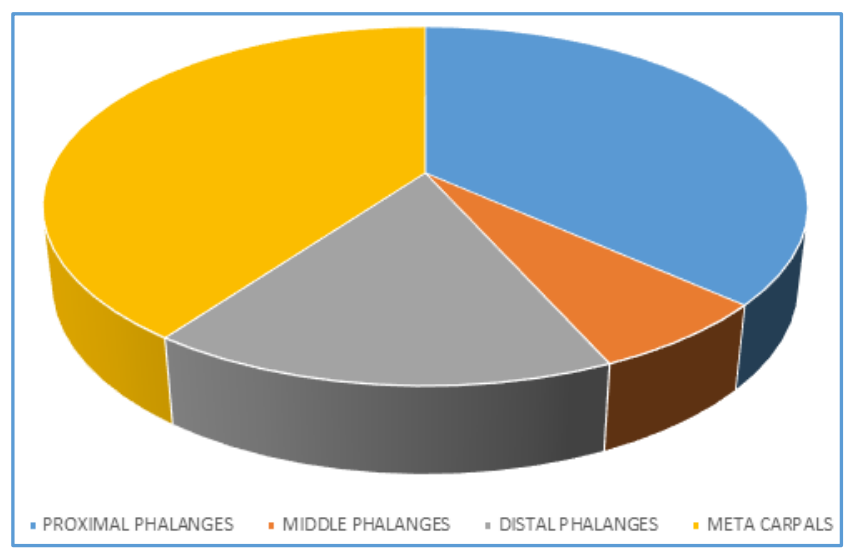

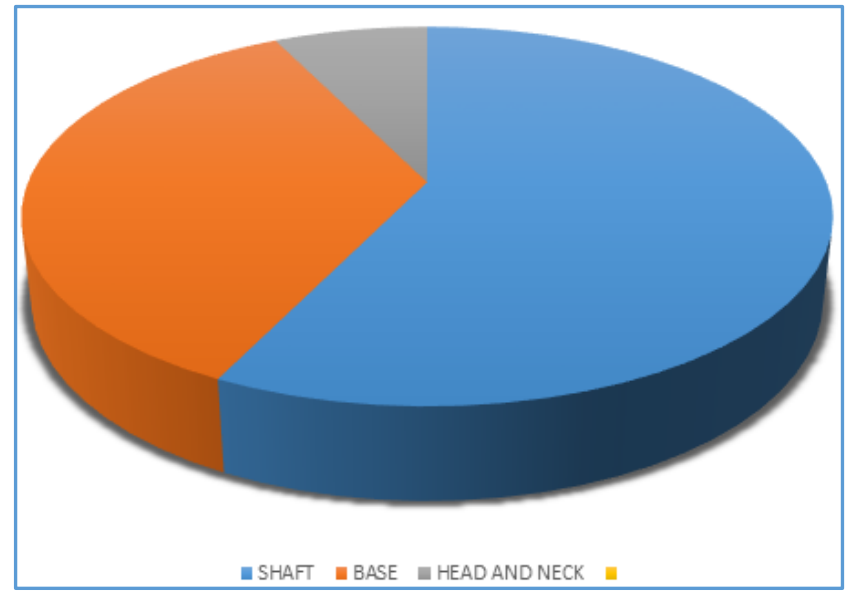

Fig. 2: Graph showing Distribution of the Sample by Location of the Fracture

Shaft fractures of metacarpal and phalanx were common (55\%) followed by base (38\%), followed by equal percentage of head and neck fractures (7\%) each.

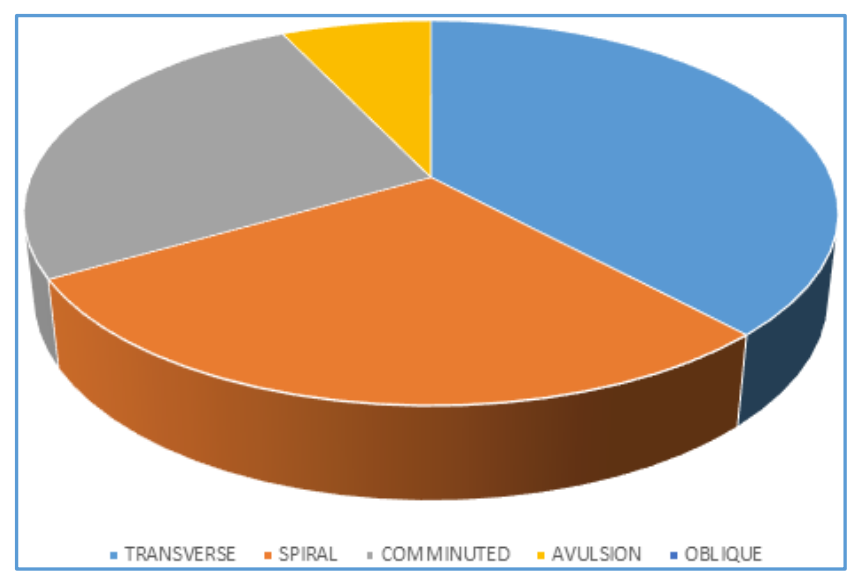

Fig. 3: Graph showing the Distribution of the Sample by the Type of the Fracture

In this study $36.36 \%$ of fractures were transverse, $27.30 \%$ were spiral, $25 \%$ were comminuted, $4.54 \%$ were oblique and $6.80 \%$ were avulsion type.

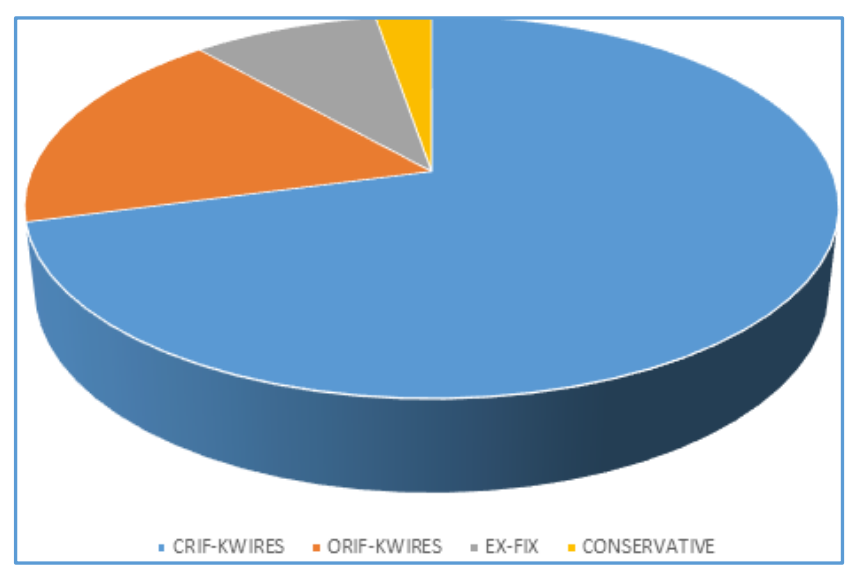

Fig. 4: Graph showing Distribution of the Sample by the Various Modalities of Treatment

Fig. 1: Graph showing Distribution of Sample by Anatomical Site 
In this study 32 cases were treated with CRIF - K-wires, 8 with ORIF - K-wires, 4 Ex-Fix, and 16 conservative management.

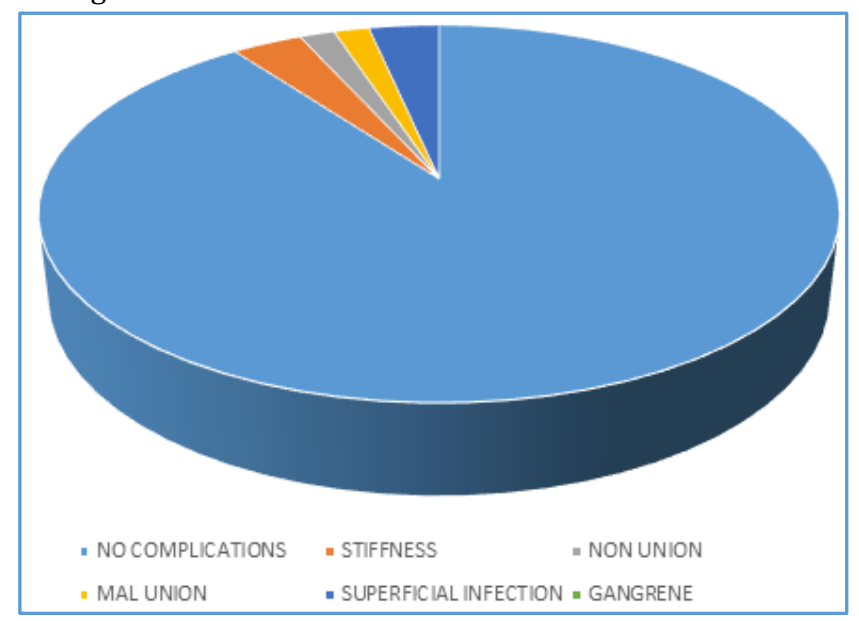

Fig. 5: Graph showing Distribution of the Sample by the Complications

In this study of 60 patients (89\%) had no complication, 2 (3.55\%) had stiffness, and 1 had mal-union (Conservatively managed), 1 had non-union (CRIF - K-Wires), 1 gangrene (ORIF - K-Wires) and 2 had superficial infection.

\section{DISCUSSION}

Transverse fractures were the most commonly observed pattern in this study, i.e. 22 cases (36.36\%), of which 16 of shaft and 6 cases of neck were transverse type followed by spiral fractures which included 16 cases (27.3\%). In a study of 35 patients by Hemant Patankar et al $1,40 \%$ of fractures were transverse, $34 \%$ short oblique, $20 \%$ long oblique and $6 \%$ were segmental. Among the many surgical methods open reduction and intramedullary K-wire osteosynthesis of metacarpal bones stands out because of the simplicity of the method and the fact it puts least strain on sliding tissue, however, stiffness of finger were much higher. In our study out of 60 cases, 8 patients (were treated with ORIF with K-wire fixation. Fifth metacarpal/phalanx were most commonly involved in this series, 18 cases (29.54\%) had 5th metacarpal/phalanx fracture, $16(27.27 \%)$ patients had involvement of $3 \mathrm{rd}$ metacarpal/phalanx fracture as compared to the study of 701 fractures by Stanton $\mathrm{J}$ S, et al, ${ }^{3}$ frequency of the fingers injured were little $(32 \%)$ followed by thumb (23\%), ring, middle and index.

In this study, metacarpal phalangeal shaft was involved in 33 cases $(55.09 \%) ; 23(38.02 \%)$ cases were base fractures as compared to studies by Gupta $\mathrm{R}$, et $\mathrm{al}^{2}{ }^{2}$ shaft fracture constituted $60 \%$. In the study of 701 fractures of the tubular bones of the hand by Stanton J et al, 3 the location of fracture was in base in $44 \%$, in shaft in $29 \%$, in neck in $17 \%$ and in head in $10 \%$. Closed reduction with subsequent intramedullary Kwire (CRIF) under image intensifier produce good functional result in long term. With low rate of complication CRIF with Kwire under image intensifier can be recommended for stabilization of metacarpal and phalangeal fracture and implant removal at outpatient department is the further advantage.

In our study 33 patients (54\%) had excellent result, 19 patients (31\%) with good result and remaining 8 patients (13\%) had fair result as comparable to other studies by Page and Stern (Excellent 62\%, Good 14\%, Fair 13\%), Duncan (Excellent 40\%, Good 24\%, Fair 7\%), Drenth and Klasen (Excellent 35\%, Good 34\%, Fair 10\%) and Gupta et al (Excellent 47\%, Good 47\%, Fair 7\%). Although, in this study sample size is small (60 cases) with low rate of complication CRIF with K-wire under image intensifier can be recommended for stabilization of metacarpal and phalangeal fractures; intra-operative image intensifier is the only drawback and implant removal at outpatient department is the further advantage.

\section{CONCLUSION}

The present study involved 60 patients with metacarpal and phalangeal fractures treated surgically with mini external fixator and K-wires. The study was conducted at Bowring and Lady Curzon Hospital and Victoria Hospital, Department of Orthopaedics, which is attached to Bangalore Medical College and Research Institute, Bangalore. The study was done from Oct. 2013 to Sep. 2015. Transverse fractures were the most commonly observed pattern in this study, 22 in total of which 16 were shaft and 6 were neck fractures. In the study, out of 60 cases 8 patients $(6.81 \%)$ were treated with ORIF with Kwire fixation. In the study, 2 patients treated with ORIF with K-wire fixation had superficial infection, which was treated with appropriate antibiotics and regular dressing.

In this study 33 patients (54\%) had excellent result, 19 patients (31\%) with good results and remaining 8 patients (13\%) had fair result? No purely implant related complications were noted (viz. K-wire loosening, breakage, migration). We suggested that metacarpal and phalangeal fractures treated by K-wires and mini external fixators gives good results than those treated conservatively in terms of union, recovery of daily activities especially in cases of displaced and multiple fractures. However, stiffness of fingers is of major concern and physiotherapy plays a major role to combat stiffness. With low rate of complication CRIF with Kwire under image intensifier can be used for stabilization of metacarpal fracture, intra-operative image intensifier, radiation is the only drawback and implant removal at outpatient department is the further advantage.

\section{REFERENCES}

1. Hemant Patankar, Fayaz W Meman. Multiple intramedullary nailing of proximal phalangeal fractures of hand. Indian J Ortho 2008;42(3):342-47.

2. Gupta R, Singh R, Siwach RC, et al. Evaluation of surgical stabilization of metacarpal and phalangeal fractures of hand. Indian J Ortho 2007;41(3):224-29.

3. Stanton JS, Dias JJ, Burke FD. Fractures of the tubular bones of the hand. J Hand Surgery 2007;32(6):626-36. 